\title{
The mechanism regulating the dissociation of the centrosomal protein C-Nap1 from mitotic spindle poles
}

\author{
Thibault Mayor ${ }^{1, \star}$, Ulrike Hacker ${ }^{1}$, York-Dieter Stierhof ${ }^{2}$ and Erich A. Nigg ${ }^{1, \neq}$ \\ ${ }^{1}$ Department of Cell Biology, Max-Planck-Institute for Biochemistry, Am Klopferspitz 18a, D-82152 Martinsried, Germany \\ 2Department of Membrane Biology, Max-Planck-Institute for Biology, Corrensstrasse 38, D-72076 Tübingen, Germany \\ *Present address: Division of Biology, California Institute of Technology, 1200 E. California Blvd, Pasadena, CA 91125, USA \\ ‡Author for correspondence (e-mail: nigg@biochem.mpg.de) \\ Accepted 26 May 2002 \\ Journal of Cell Science 115, 3275-3284 (2002) (C) The Company of Biologists Ltd
}

\section{Summary}

The centrosomal protein $\mathrm{C}$-Nap1 is thought to play an important role in centrosome cohesion during interphase of the cell cycle. At the onset of mitosis, when centrosomes separate for bipolar spindle formation, C-Nap1 dissociates from centrosomes. Here we report the results of experiments aimed at determining whether the dissociation of C-Nap1 from mitotic centrosomes is triggered by proteolysis or phosphorylation. Specifically, we analyzed both the cell cycle regulation of endogenous $\mathrm{C}$-Nap1 and the fate of exogenously expressed full-length C-Nap1. Western blot analyses suggested a reduction in the endogenous $\mathrm{C}$-Nap1 level during $M$ phase, but studies using proteasome inhibitors and destruction assays performed in Xenopus extracts argue against ubiquitin-dependent degradation of $\mathrm{C}$-Nap1. Instead, our data indicate that the mitotic $C$-Nap1 signal is reduced as a consequence of $M$ phase-specific phosphorylation. Overexpression of full- length C-Nap1 in human U2OS cells caused the formation of large structures that embedded the centrosome and impaired its microtubule nucleation activity. Remarkably, however, these centrosome-associated structures did not interfere with cell division. Instead, centrosomes were found to separate from these structures at the onset of mitosis, indicating that a localized and cell-cycle-regulated activity can dissociate $\mathrm{C}$-Nap1 from centrosomes. A prime candidate for this activity is the centrosomal protein kinase Nek2, as the formation of large C-Nap1 structures was substantially reduced upon co-expression of active Nek2. We conclude that the dissociation of $\mathrm{C}$-Nap1 from mitotic centrosomes is regulated by localized phosphorylation rather than generalized proteolysis.

Key words: C-Nap1, Nek2, Centrosome, Mitotic spindle, Phosphorylation

\section{Introduction}

The centrosomal protein C-Nap1, also known as Cep250 (Mack et al., 1998) has been implicated in the cell-cycleregulated cohesion of microtubule-organizing centers (Fry et al., 1998a; Mayor et al., 2000). This $281 \mathrm{kDa}$ protein consists mainly of domains predicted to form coiled coil structures. During interphase, C-Nap1 localizes to proximal ends of both parental centrioles, but it dissociates from these structures at the onset of mitosis. Re-association with centrioles then occurs in late telophase or at the very beginning of G1 phase, when daughter cells are still connected by post-mitotic bridges (Mayor et al., 2000). Electron microscopic studies performed on isolated centrosomes suggest that a proteinaceous linker connects parental centrioles (Paintrand et al., 1992). Considering the localization of C-Nap1 (Fry et al., 1998a; Mayor et al., 1999), it is attractive to speculate that C-Nap1 constitutes a docking site for such a linker, and this view is supported by functional studies. Microinjection of anti-C-Nap1 antibodies into tissue culture cells induces centrosome splitting (i.e. the separation of parental centrioles by a distance of several microns), and centrosome splitting can also be induced by overexpression of C-Nap1 truncation mutants that presumably exert a dominant-negative effect (Mayor et al.,
2000). Thus, a current model holds that C-Nap1 is part of a linker structure that assures the cohesion of duplicated centrosomes during interphase, but that is dismantled upon centrosome separation at the onset of mitosis.

C-Nap1 is known to interact with the mammalian serine/ threonine kinase Nek2 (Fry et al., 1998a), a member of the NIMA kinase family (Schultz et al., 1994). Both Nek2 abundance and activity are cell cycle regulated, with peak levels in S/G2 and low activity in nocodazole-arrested and G1 phase cells (Fry et al., 1995). Both C-Nap1 and Nek2 colocalize at the centrosome (Fry et al., 1998a), and C-Nap1 most likely constitutes a physiological substrate of Nek2 (Fry et al., 1998a). Interestingly, protein phosphatase 1 (PP1) interacts with Nek2 and can de-phosphorylate both C-Nap1 and Nek2 (Helps et al., 2000). Thus, it is attractive to speculate that phosphorylation may regulate $\mathrm{C}-\mathrm{Nap} 1$ function, and that the phosphorylation state of C-Nap1 may depend on the balance of Nek2 and PP1 activity. In support of such a model, centrosome splitting can be induced by overexpression of active Nek2 (Fry et al., 1998b) as well as by drug-induced inhibition of PP1 (Meraldi and Nigg, 2001). From a cell cycle perspective, it is intriguing that PP1 is inhibited at the onset of mitosis, possibly as a direct consequence of phosphorylation 
by Cdk1 (Puntoni and Villa-Moruzzi, 1997). As a result, Nek2 is expected to prevail and phosphorylate C-Nap1 specifically at the onset of mitosis.

Although attractive, the above model remains speculative. In particular, there is no previous evidence to indicate that endogenous C-Nap1 is phosphorylated in vivo, let alone that it is phosphorylated in a cell-cycle-specific manner. Furthermore, there is increasing evidence to suggest that ubiquitin-dependent proteolysis of centrosomal proteins plays an important role in the regulation of centrosome structure and function (Freed et al., 1999; Gstaiger et al., 1999; Fabunmi et al., 2000). In the present study, we thus asked whether proteolysis or phosphorylation is more likely to explain the cell-cycleregulated dissociation of C-Nap1. To this end, we studied both the cell cycle regulation of endogenous C-Nap1, and the consequences of overexpressing full-length exogenous CNap1. Our results provide no evidence for proteolytic degradation of C-Nap1. Instead, they strengthen the view that C-Nap1 localization and function is regulated primarily by cell-cycle-regulated phosphorylation.

\section{Materials and Methods}

\section{Plasmid construction}

For construction of truncated versions of myc-tagged C-Nap1 (T3-7), the ${ }^{\mathrm{k}} \mathrm{C}$-Nap1 plasmid (Mayor et al., 2000) was used. During these constructions, linker sequences were generated both between the myc tag and the C-Nap1 fragment, and upstream of the stop codon. These sequences, as well as the amino acid (aa) sequence of the corresponding C-Nap1 mutants, are as follows: T3 (aa 243-1985; MNSCSPSRA, DS), T4 (aa 1-1986; MNSCSPSRA, DR), T5 (aa 11182; MNSCSPSRA, GGIH), T6 (aa 1248-2442; MNSCSPGDQ, -), T7 (aa 243-2442;MNSCS; -). All mutant constructs were subcloned into the pBK-CMV vector. For fusion to the enhanced GFP marker, C-Nap1 was excised from the ${ }^{\mathrm{k}} \mathrm{C}-\mathrm{Nap} 1$ plasmid using EcoRV and SacII and introduced into the sites BspEI (blunted with Klenow) and SacII of pEGFP-C1 (Clontech), creating a 6 aa linker (SGSRAA). The GFP-C-Nap1 fusion protein was further subcloned into pcDNA4/TO (Invitrogen) using, respectively, AgeI (blunted with Klenow) and BamHI, and HindIII (blunted with Klenow) and BamHI.

\section{Cell culture}

$\mathrm{HeLa}$ and U2OS osteosarcoma cells were grown at $37^{\circ} \mathrm{C}$ in a $7 \%$ or $5 \% \mathrm{CO}_{2}$ atmosphere in Dulbecco's modified Eagle's medium (DMEM) supplemented with $10 \%$ heat-inactivated fetal calf serum (FCS) and penicillin-streptomycin (100 i.u./ml and $100 \mu \mathrm{g} / \mathrm{ml}$, respectively). HeLa cells were treated with $2 \mathrm{mM}$ thymidine, 1.6 $\mu \mathrm{g} / \mathrm{ml}$ aphidicolin, $0.5 \mu \mathrm{g} / \mathrm{ml}$ nocodazole, $100 \mu \mathrm{M}$ monastrol, 10 or $40 \mu \mathrm{g} / \mathrm{ml} \mathrm{MG132} \mathrm{(C-2211),} 10$ or $25 \mu \mathrm{g} / \mathrm{ml}$ MG115 (C-6706) or $5 \mu \mathrm{g} / \mathrm{ml}$ ALLN (A-6185). (Monastrol was a kind gift from $\mathrm{Dr} \mathrm{T}$. Mayer, all other drugs from Sigma.) For synchronization at G1/S, dishes with $20 \%$ confluent cells were treated for 14 hours with thymidine, washed three times with PBS, reincubated with normal medium for 10 hours and, finally, treated for 14 hours with aphidicolin. For synchronization in mitosis, cells were released for 10 hours from the G1/S block, treated with nocodazole or monastrol for 4.5 hours and collected by mechanical shake off. Alternatively, mitotic cells were collected by mechanical shake off after 16 hours of nocodazole treatment. For synchronization in G1, cells blocked with nocodazole in mitosis were collected, washed and released for 6 hours into normal medium. Mechanical shake off was then applied to discard non-adherent cells.

For transient transfection studies, U2OS cells were seeded onto $\mathrm{HCl}$-treated glass coverslips and then transfected with $5 \mu \mathrm{g}$ of plasmid
DNA, using calcium phosphate precipitates as previously described (Seelos, 1997). Cells were fixed 24 or 36 hours later in cold methanol $\left(-20^{\circ} \mathrm{C}\right)$ for 6 minutes. To arrest cells in mitosis, a mutated chicken cyclin B2 in pCMV-neo was used (arginine in position 32 converted to serine, Gallant and Nigg, 1992). Co-transfection was achieved by adding $2.5 \mu \mathrm{g}$ of each of the two plasmid DNAs. When active (wt) and inactive Nek2 kinases in pRc-CMV (Fry et al., 1998b) were cotransfected with mycC-Nap1, $3.5 \mu \mathrm{g}$ and $1.5 \mu \mathrm{g}$ of plasmid DNA were used for Nek2 and C-Nap1, respectively.

Enhanced GFP-C-Nap1 was stably integrated into T-REx modified U2OS cells according to manufacturer's instructions (Invitrogen), and cells were cloned by limiting dilution. Expression of the GFP-C-Nap1 fusion protein was induced for 24 hours with $1 \mu \mathrm{g} / \mathrm{ml}$ of tetracycline (Invitrogen, Q100-19).

\section{Immunofluorescence microscopy}

Cells were washed with PBS and blocked for 20 minutes with $1 \%$ BSA in PBS at room temperature, before being incubated with primary antibodies for 1 hour at room temperature. Primary antibodies were affinity-purified anti-C-Nap1 (C-Ab, $0.5 \mu \mathrm{g} / \mathrm{ml})$, GTU-88 anti$\gamma$-tubulin mAb (1:1000; Sigma), anti- $\alpha$-tubulin mAb (1:2000; Sigma), 9E10 anti-myc mAb (undiluted tissue culture supernatant) and GT335 anti-polyglutamylated tubulin mAb (1:2000; a kind gift of B. Edde, CNRS, Montpellier, France). Secondary antibodies were biotinylated donkey anti-rabbit or goat anti-mouse IgG (1:200; Amersham), followed by Texas-Red-conjugated streptavidin (1:100; Amersham), FITC-conjugated goat anti-mouse Fab fragment (1:100; Sigma), FITC-conjugated goat anti-rabbit (1:100; Sigma) and Alexa-Fluor488-conjugated goat anti-mouse IgG (1:1000; Molecular Probes). All antibodies were diluted in 3\% BSA in PBS. Immunofluorescence microscopy was performed using a Zeiss Axioplan II microscope and Neofluar 40× or Apochromat $63 \times$ oil immersion objectives. Photographs were taken using Quantix 1400 (Photometrics) or Micromax (Princeton Instruments) CCD cameras and IP-Lab or Metaview (Universal Imaging) software.

\section{Immunochemical techniques}

To prepare extracts for immunoblotting, cells were lysed in RIPA buffer (50 mM Tris- $\mathrm{HCl}, \mathrm{pH} 8,1 \%$ Nonidet $\mathrm{P} 40,0.5 \%$ deoxycholic acid, $0.1 \%$ SDS, $150 \mathrm{mM} \mathrm{NaCl}, 1 \mathrm{mM}$ PMSF, $1 \mu \mathrm{g} / \mathrm{ml}$ aprotinin, 1 $\mu \mathrm{g} / \mathrm{ml}$ leupeptin, $1 \mu \mathrm{g} / \mathrm{ml}$ pepstatin A, $20 \mathrm{mM} \beta$-glycerophosphate, 20 $\mathrm{mM} \mathrm{NaF}$ and $0.3 \mathrm{mM}$ sodium vanadate). Samples were left for 30 minutes on ice, passed 10 times through a $27 \mathrm{G}$ needle and centrifuged at $16,000 \mathrm{~g}$ for 10 minutes at $4{ }^{\circ} \mathrm{C}$. Protein concentrations were determined using an improved Lowry assay ( $D c$ protein assay, Biorad). C-Nap1 antibodies were rabbit polyclonal C-Ab (aa 19822442) and M-Ab (aa 1098-1248) (Fry et al., 1998a; Mayor et al., 2000). All antibody incubations were carried out in blocking buffer (5\% nonfat dried milk in PBS/0.1\% Tween-20) at the following dilutions: C-Ab, $0.5 \mu \mathrm{g} / \mathrm{ml} ; \mathrm{M}-\mathrm{Ab}, 1 \mu \mathrm{g} / \mathrm{ml}$; anti-cdc27 serum, 1/1000; anti-cyclin A, 1/500; anti- $\alpha$-tubulin mAb, 1/1000 (Sigma); anti-GFP mAb, undiluted tissue culture supernatant. Bound IgGs were visualized using alkaline phosphatase-conjugated anti-rabbit or antimouse IgG secondary antibodies (1:7500; Promega).

\section{Alkaline phosphatase treatment}

HeLa cells were lysed in IP buffer (0.5\% Igepal, $150 \mathrm{mM} \mathrm{NaCl}, 50$ $\mathrm{mM}$ Tris-HCl pH 8, $20 \mathrm{mM} \beta$-glycerophosphate, $20 \mathrm{mM} \mathrm{NaF}$ and 0.3 $\mathrm{mM} \mathrm{NaVO} 4$ ). Samples containing equal amounts of proteins were mixed for 1 hour at $4^{\circ} \mathrm{C}$ with $\mathrm{C}-\mathrm{Ab}(8 \mu \mathrm{g} / \mathrm{ml})$ pre-bound to Affiprep protein $\mathrm{A}$ beads $(1 \mu \mathrm{l} / \mu \mathrm{g}$ of $\mathrm{Ab}$; Biorad). Beads were washed three times with IP buffer and resuspended in $2 \times 200 \mu \mathrm{l}$ of AP buffer $(10$ $\mathrm{mM}$ Tris-HCl, $10 \mathrm{mM} \mathrm{MgCl} 2,50 \mathrm{mM} \mathrm{NaCl}, 1 \mathrm{mM}$ DTT, pH 7.9, with $1 \mathrm{mM}$ PMSF, $1 \mu \mathrm{g} / \mathrm{ml}$ aprotinin, $1 \mu \mathrm{g} / \mathrm{ml}$ leupeptin and $1 \mu \mathrm{g} / \mathrm{ml}$ 
pepstatin). Reactions were then carried out by incubating these 200 $\mu \mathrm{l}$ aliquots for 40 minutes at $37^{\circ} \mathrm{C}$ with 20 units of either active or heat-inactivated $\left(10\right.$ minutes at $\left.95^{\circ} \mathrm{C}\right)$ calf intestinal alkaline phosphatase (M0290; New England BioLabs). Finally, beads were resuspended in sample buffer.

In vivo [32P] labeling

HeLa cells in G1 were collected 4 hours after release from a 16 hour nocodazole block. Cells in S, G2 and M phase were obtained after releases for 2, 6 or 10 hours, respectively, from a double-block with thymidine-aphidicolin. M-phase cells were further blocked with nocodazole for an additional 5 hours and then collected by mechanical shake-off. All cells were labeled by additional incubation for 4 hours in phosphate-free DMEM medium that had been supplemented with $10 \%$ dialyzed FCS, 10\% normal DMEM, 1\% normal FCS and 400 $\mu \mathrm{Ci} / \mathrm{ml}$ of phosphoric acid ( $1 \mathrm{mCi}$ in total). Cells were collected and lysed in $400 \mu \mathrm{1} / 2 \times 10^{6}$ cells of modified-IP buffer (0.4\% Igepal, $0.1 \%$ deoxycholic acid, $150 \mathrm{mM} \mathrm{NaCl}, 50 \mathrm{mM}$ Tris- $\mathrm{HCl} \mathrm{pH}$ 8, $20 \mathrm{mM} \beta$ glycerophosphate, $20 \mathrm{mM} \mathrm{NaF}$ and $0.3 \mathrm{mM} \mathrm{NaVO}_{4}$ ). Orthophosphate incorporation in the various cell extracts was measured by scintillation counting, and equal protein levels were confirmed by Coomassie blue staining. Samples were then pre-cleared using rabbit IgG bound to Protein A-Dynabeads (Dynal, $10 \mu \mathrm{g}$ IgG to $40 \mu \mathrm{l}$ beads/400 $\mu \mathrm{l}$ extract), and immunoprecipitations were performed with $\mathrm{C}-\mathrm{Ab}$ (and rabbit IgG for control) bound to Protein A-Dynabeads pre-blocked with HeLa cell extracts ( $5 \mu \mathrm{g} \mathrm{IgG}$ to $20 \mu \mathrm{l}$ beads/200 $\mu \mathrm{l}$ extract). To ensure low background, samples were resuspended in sample buffer $(30 \mu \mathrm{l})$, heated for 5 minutes at $95^{\circ} \mathrm{C}$, diluted in $1 \mathrm{ml}$ of modified-IP buffer and subjected to a second round of immunoprecipitation.

\section{Miscellaneous techniques}

Immunoelectron microscopy was performed as described previously (Fry et al., 1998a). For assaying protein degradation, cyclin B2 and myc-C-Nap1 were in vitro translated, in the presence of $\left[{ }^{35} \mathrm{~S}\right]-\mathrm{Met}$ (TNT coupled reticulocyte system, Promega), and added to M-phase Xenopus egg extracts ( $12.5 \%$ of final volume). These were prepared by complementing interphasic extracts with recombinant $\Delta 90$-cyclin $\mathrm{B}$ and cycloheximide $(0.25 \mu \mathrm{g} / \mu \mathrm{l})$, followed by a 90 minute incubation at $22^{\circ} \mathrm{C}$ (Vorlaufer and Peters, 1998). Aliquots were removed every 15 minutes, mixed with sample buffer and heated for 5 minutes at $95^{\circ} \mathrm{C}$.

\section{Results}

\section{Cell cycle analysis of C-Nap1 protein levels}

To determine whether C-Nap1 levels are regulated during the cell cycle, HeLa cells were synchronized at different cell cycle stages. Equal amounts of protein were then resolved by SDSPAGE and analyzed by western blotting. Compared with asynchronous, G1 or G2 cells, the C-Nap1 signal was significantly diminished in the M-phase sample (Fig. 1A). This reduction in C-Nap1 signal was highly reproducible and independent of the antibody used for detection (data not shown, but see Fig. 1C and Fig. 2A). Equal sample loading was confirmed by western blotting with $\alpha$-tubulin antibodies (Fig. 1A) and Coomassie blue staining (data not shown). As a direct control for the M-phase state, western blotting was also performed with antibodies against Cdc27, a subunit of the APC/cyclosome complex that displays a reduced electrophoretic mobility upon M-phase-specific phosphorylation (Fig. 1A). Cell cycle arrest experiments were also performed using monastrol. This drug inhibits the kinesinrelated motor Eg5 and thus arrests cells in early mitosis with monopolar spindles, without disturbing MTs (Mayer et al., 1999; Kapoor et al., 2000). As shown in Fig. 1B, the C-Nap1 protein signal was reduced to a similar extent, regardless of whether cells had been arrested with monastrol or nocodazole. Furthermore, no C-Nap1 could be detected in any of the insoluble fractions, arguing against cell cycle variability in CNap1 extractability (Fig. 1B). Finally, mitotic cells were also collected by mechanical shake off, and samples analyzed by western blotting using antibodies directed against either the $\mathrm{C}$ terminus $(\mathrm{C}-\mathrm{Ab})$ or the central domain $(\mathrm{M}-\mathrm{Ab})$ of $\mathrm{C}-\mathrm{Nap} 1$. In all these experiments, the C-Nap1 signal was reduced by about $30-40 \%$ in M-phase cells when compared with asynchronously growing cells (Fig. 1C).
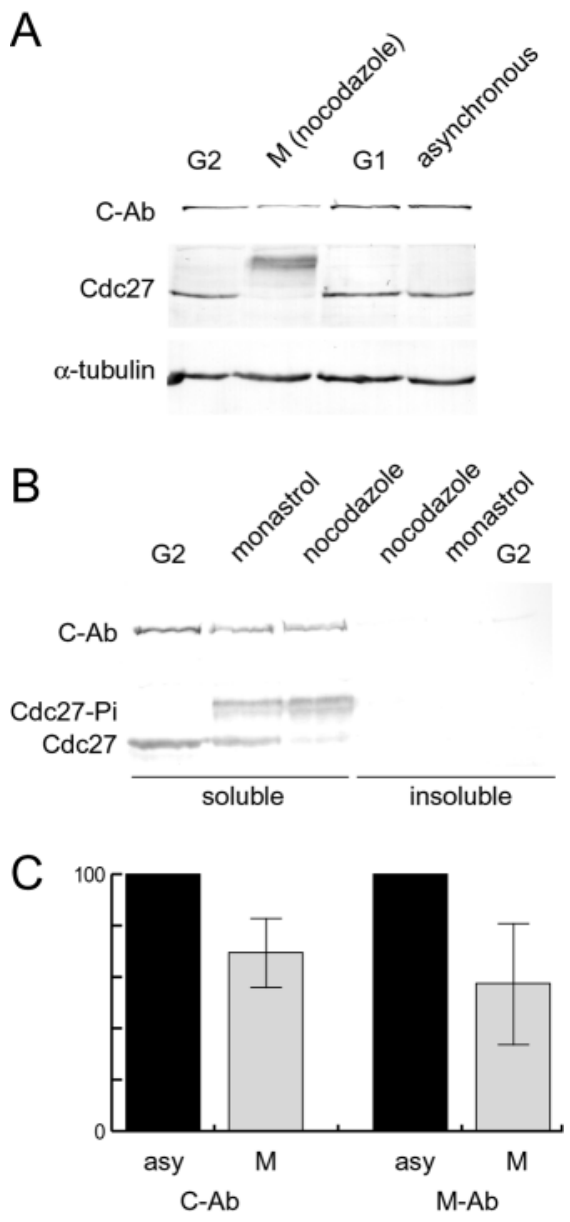

Fig. 1. Loss of C-Nap1 staining during mitosis. (A) HeLa cells were synchronized at the indicated cell cycle stages. Equal amounts of protein were then separated by $10 \%$ SDS-PAGE and probed by immunoblotting with the antibodies indicated. (B) HeLa cells were collected in G2 or blocked in mitosis using either nocodazole or monastrol, as indicated. Equal amounts of protein were separated by $7.5 \%$ SDS-PAGE, and probed by immunoblotting. For the control, insoluble fractions were also resuspended and corresponding volumes (1:1) analyzed in parallel. (C) Mitotic HeLa cells (M) were collected by mechanical shake off and asynchronously growing cells (asy) by trypsinization. Levels of C-Nap1 were then determined by immunoblotting, using either C-Ab or M-Ab (Fry et al., 1998a; Mayor et al., 2000). All C-Nap1 signals were standardized relative to the $\alpha$-tubulin signal. The histogram shows the relative levels of $\mathrm{C}$ Nap1 in M-phase and asynchronously growing cells. 
The above results suggested that C-Nap1 could be subject to cell-cycle-regulated proteolytic degradation. To determine whether the inhibition of the proteasome would result in a stabilization of C-Nap1, M-phase arrest experiments were performed in the presence of increasing amounts of the proteasome inhibitor MG132. As shown in Fig. 2A, C-Nap1 levels were reduced even in the presence of this inhibitor. In contrast, the proteasome-target cyclin A was stabilized, as expected, demonstrating the efficacy of proteasome inhibition (Fig. 2A). Virtually identical results were obtained using the proteasome inhibitors MG115 and N-acetyl-leucyl-leucylnorleucinal (ALLN) (data not shown). In principle, C-Nap1 could be a substrate for other proteases, but we emphasize that no distinct cleavage products could ever be observed in any of the above experiments. To more directly assess C-Nap1 stability during M phase, we turned to Xenopus egg extracts, which provide a convenient assay system for examining protein degradation (Vorlaufer and Peters, 1998). When $\left[{ }^{35} \mathrm{~S}\right]$-labeled, in vitro translated C-Nap1 and cyclin B were added to such extracts, C-Nap1 was completely stable, whereas cyclin B was almost totally degraded after 60 minutes of incubation (Fig. 2B). Taken together, these results indicate that C-Nap1 is not subject to degradation by the proteasome, and they also argue against cleavage of C-Nap1 by other M-phase-specific proteases.

\section{Evidence for M-phase-specific phosphorylation of C-Nap1}

As the fate of mitotic C-Nap1 did not seem to be governed by
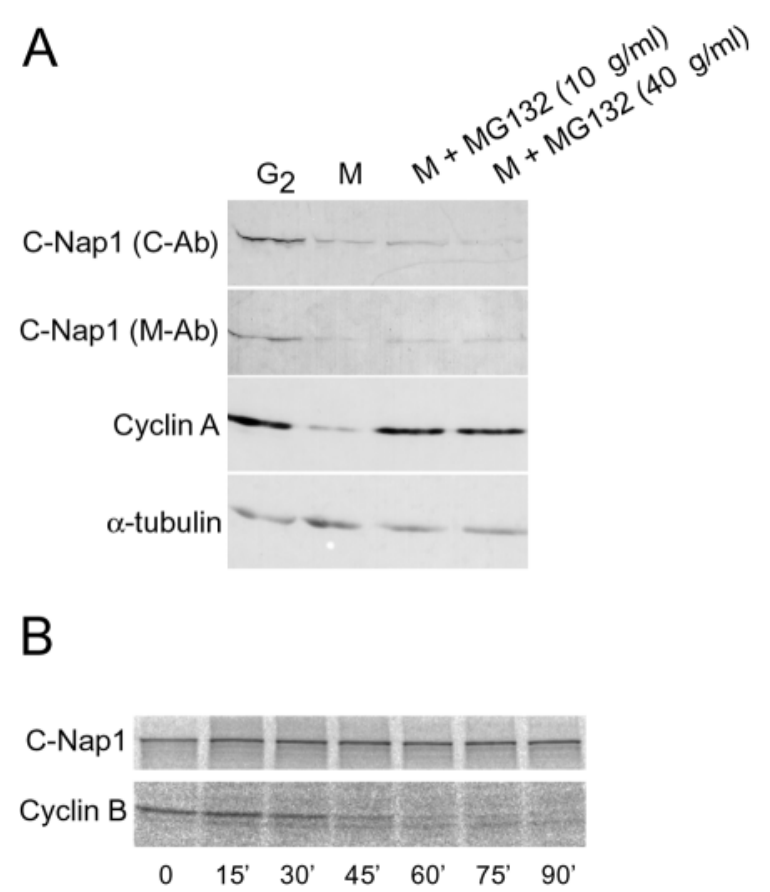

Fig. 2. C-Nap1 is not degraded during mitosis. (A) HeLa cells were collected in $\mathrm{G} 2$ or blocked in mitosis in the presence or absence of the proteasome inhibitor MG132. Equal amounts of protein were separated by $7.5 \%$ SDS-PAGE and probed by immunoblotting using the antibodies indicated. (B) Destruction assay in Xenopus egg extract. $\left[{ }^{35} \mathrm{~S}\right]$-labeled cyclin B and C-Nap1 were added to a mitotic extract and samples taken at the indicated times (minutes). Proteins were separated by $10 \%$ SDS-PAGE and exposed for autoradiography. proteolytic degradation, we next considered the possibility that the M-phase-specific reduction in the C-Nap1 signal could reflect a post-translational modification. In particular, we reasoned that M-phase-specific phosphorylation might have caused an altered, more diffuse electrophoretic mobility of CNap1, or a reduced transfer to nitrocellulose membranes. To explore this possibility, C-Nap1 was immunoprecipitated from extracts at different cell cycle stages, and then treated with calf intestinal phosphatase prior to western blotting. As shown in Fig. 3A, the staining of mitotic C-Nap1 was increased in response to active phosphatase but not the heat-inactivated enzyme. Similar results were obtained when mitotic cell extracts were incubated at $37^{\circ} \mathrm{C}$ in the absence of phosphatase inhibitors (data not shown). These data show that a nearinterphasic C-Nap1 signal could be recovered by treatment of mitotic C-Nap1 with an active phosphatase, implying that CNap1 was phosphorylated when isolated from mitotic cells.

To directly demonstrate that C-Nap1 is phosphorylated during mitosis, in vivo labeling with $\left[{ }^{32} \mathrm{P}\right]$ phosphoric acid was performed in $\mathrm{HeLa}$ cells. Extracts were then prepared from different cell cycle stages, and C-Nap1 was immunoprecipitated and analyzed by SDS-PAGE. As shown by autoradiography and western blotting, immunoprecipitated C-Nap1 was labeled with $\left[{ }^{32} \mathrm{P}\right]$ specifically during $\mathrm{M}$ phase, but not during G1, S or G2 phases (Fig. 3B). No [ $\left.{ }^{32} \mathrm{P}\right]$-labeling of C-Nap1 was observed in control immunoprecipitation experiments using nonspecific rabbit IgG (data not shown).

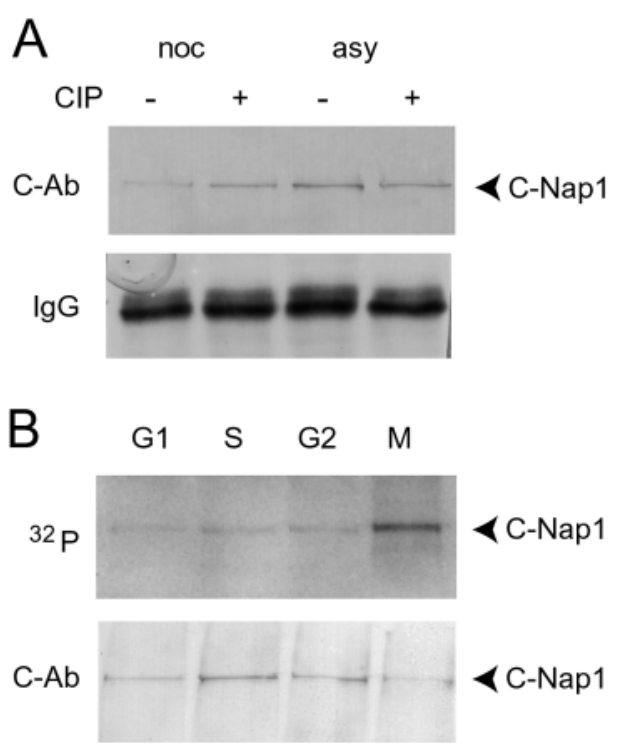

Fig. 3. C-Nap1 is phosphorylated during mitosis. (A) Alkaline phosphatase treatment enhances the western blot signal of mitotic CNap1. C-Nap1 was immunoprecipitated from asynchronously growing (asy) or nocodazole-arrested (noc) HeLa cells and treated with either active $(+)$ or heat-inactivated $(-)$ calf intestinal phosphatase (CIP). Samples were separated by 7.5\% SDS-PAGE and probed by immunoblotting as indicated (IgG are shown to demonstrate equal loading). (B) In vivo labeling of C-Nap1 with $\left.{ }^{[2} \mathrm{P}\right]$ orthophosphate. C-Nap1 was immunoprecipitated from the indicated cell cycle stages, using C-Ab. Samples were separated by 7.5\% SDS-PAGE, and gels exposed for autoradiography (top panel). In parallel, recovery of C-Nap1 was controlled by western blotting with $\mathrm{C}-\mathrm{Ab}$ (bottom panel). 
Taken together, these data indicate that C-Nap1 is indeed phosphorylated specifically during mitosis.

\section{Overexpression of C-Nap1 causes the formation of large centrosome-associated structures}

The above results strongly suggested that phosphorylation, rather than proteolysis, regulates the displacement of C-Nap1 from the centrosome at the onset of mitosis. However, because only a fraction of the total C-Nap1 protein (estimated to about $30-50 \%)$ is associated with the centrosome, it is difficult to obtain definitive information about local events occurring at the centrosome. As a complementary approach to studying the fate and regulation of C-Nap1 during the cell cycle, we thus decided to examine the phenotypic consequences of overexpressing C-Nap1 in human cells. In particular, we asked whether a high level of $\mathrm{C}$-Nap1 could prevent the dissociation of C-Nap1 from the centrosome, and thus possibly interfere with centrosome separation at the onset of mitosis. Full-length C-Nap1, with or without an N-terminal myc-tag, was therefore placed under the control of a CMV promoter and transiently transfected into U2OS cells. Then, the localization of the ectopically expressed protein was determined by immunofluorescence microscopy. In parallel, the distribution and morphology of centrosomes was examined by staining with antibodies against $\gamma$-tubulin. In cells expressing low levels of exogenous C-Nap1, the full-length protein localized correctly and almost exclusively to the centrosome (data not shown). With higher expression levels, however, cytoplasmic staining became increasingly prominent, and, most strikingly, one or several compact C-Nap1-positive aggregates (termed patches) became visible (Fig. 4A). In some cells, these patches grew into large structures occupying almost the entire cytoplasm. Immunoelectron microscopy performed on cells overexpressing C-Nap1 revealed that the centrioles were invariably embedded in large structures showing extensive labeling with anti-C-Nap1 antibodies (Fig. 4B). These structures often emanated primarily from the proximal ends of centrioles (Fig. 4Ba), but eventually extended to engulf almost the entire centrioles (Fig. 4Bb).

To further characterize the relationship between the observed C-Nap1 patches and centrosomes, antibodies against different centrosomal proteins were used for double immunofluorescence microscopy. Endogenous Nek2 protein was found to accumulate rather uniformly in all C-Nap1 patches (Fig. 5Ab), consistent with a direct interaction between these two proteins (Fry et al., 1998a). In contrast, the distribution of $\gamma$-tubulin appeared largely unaltered, in that anti$\gamma$-tubulin antibodies stained the typical one or two dots per cell (Fig. 5Ac). In cases where only a single C-Nap1 patch was present within a cell, this patch was invariably associated with the $\gamma$-tubulin-positive centrosome. However, if multiple patches were present, only one stained positive for $\gamma$-tubulin (Fig. 5A). Similar results were obtained for other centrosomeassociated proteins, notably centrin-2, p150 Glued and Plk1, with none of them showing a particular enrichment in the C-Nap1 patches (data not shown). These results indicate that the observed patches are composed primarily of C-Nap1 itself, and that they incorporate at most a limited subset of endogenous centrosomal proteins. Consistent with this interpretation, none of the extra-centrosomal C-Nap1 patches displayed any microtubule (MT) nucleation activity. On the contrary, a MT re-growth assay showed that centrosomes embedded in CNap1 patches displayed severely reduced MT nucleation activity (Fig. 5B). It seems plausible that this reflects steric hindrance exerted by the C-Nap1 oligomer.

In order to identify sub-domains within C-Nap1 responsible for patch formation, several truncation mutants of C-Nap1 were expressed in U2OS cells (Fig. 5C). We found that a mutant from which only the C-terminal domain had been deleted (T4) still formed extensive patches, but that further deletion of the C-terminal coiled-coil domain (T5) abolished patch formation. Furthermore, none of the mutants (T6, T7) lacking the N-terminal end domain formed patches. These data suggest that self-assembly of $\mathrm{C}$-Nap1 into oligomeric structures requires the $\mathrm{N}$-terminal end domain and both central coiled-coil domains.

\section{Overexpression of C-Nap1 does not prevent centrosome separation}

Next we sought to determine whether the overexpression of C-

Fig. 4. Overexpression of fulllength C-Nap1 causes patch formation. U2OS cells were transiently transfected with full-length C-Nap1 and, 24 hours later, processed for immunofluorescence or immunoelectron microscopy with C-Ab. For immunofluorescence microscopy (A), cells were fixed with methanol; for immunoelectron microscopy (B) cells were fixed with $3 \%$ paraformaldehyde/ $2 \%$ sucrose. C-Nap1 overexpression resulted in the formation of
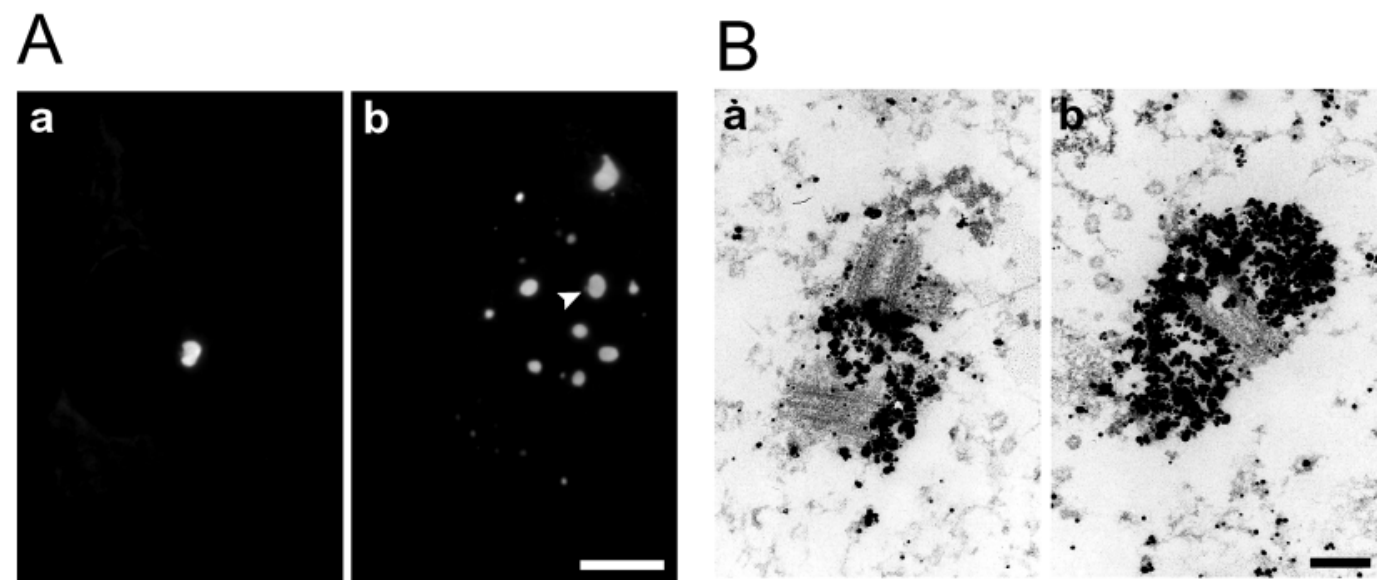
single (a) or multiple (b) patches. The arrowhead in Ab points to the patch containing the centrosome, as determined by counter-staining with anti- $\gamma$-tubulin antibodies (not shown). Bars, $10 \mu \mathrm{m}(\mathrm{Ab}) ; 250 \mathrm{~nm}(\mathrm{Bb})$. 
Nap1 would deregulate centrosome separation and/or cell cycle progression. Initial experiments based on flow cytometry failed to reveal any significant differences between the cell cycle profiles of populations transfected with either CNap1 or pBK-CMV empty vector (data not shown). To ask more directly whether C-Nap1 overexpression would prevent entry of cells into mitosis, C-Nap1 was coexpressed with a non-destructible mutant of cyclin B2, which is known to cause the accumulation of mitotic cells (Gallant and Nigg, 1992). If the observed embedding of centrosomes into large C-Nap1 patches was to block entry of cells into mitosis, coexpression of C-Nap1 should have suppressed the accumulation of mitotic cells. However, this was not observed: upon co-expression of C-Nap1 with nondestructible cyclin B2, about $50 \%$ of the transfected cells arrested in mitosis (Fig. 6A). This extent of mitotic arrest was similar to that seen in control experiments, in which non-destructible cyclin B2 was co-transfected with the enhanced GFP marker alone (Fig. 6A). Conversely, the accumulation of mitotic cells was severely suppressed upon co-expression of the nondestructible cyclin B2 with a dominantnegative Cdk1 mutant. As this Cdk1 mutant is known to cause a G2 arrest (van den Heuvel and Harlow, 1993), this result validates our experimental approach and establishes that overexpression of C-Nap1 does not prevent cells from entering mitosis.

From the above results it is difficult to escape the conclusion that cells were able to undergo mitosis in spite of the fact that their interphase centrosomes were embedded in large C-Nap1 structures. This begs the question of how such embedded centrosomes could separate and contribute to the formation of a functional mitotic spindle. To address this issue, we established a U2OS cell line stably expressing a full-length GFP-C-Nap1 fusion protein under tetracycline control. Within 24 hours of tetracycline addition, exogenous GFP-C-Nap1 was readily expressed, as demonstrated by western blotting (Fig. $6 \mathrm{~B})$. When analyzed by fluorescence microscopy, GFP-CNap1 formed patches that were indistinguishable from those described above (data not shown, but see below). The stably transformed U2OS cells were then grown for 6 days in the presence or absence of tetracycline, and their growth curves determined. In a third U2OS cell population, GFP-C-Nap1 expression was pre-induced for 24 hours, before the culture was continued for 6 days in the presence of tetracycline. As shown in Fig. 6C, all three cell lines showed indistinguishable proliferation rates, confirming that cells proliferate normally in the presence of extensive C-Nap1 patches. To further test this surprising conclusion, we directly examined spindle formation in cells harboring C-Nap1 patches. Spindle MTs were stained with antibodies against $\alpha$-tubulin (Fig. 7A), whereas centrioles were stained with antibodies against poly-glutamylated tubulin [GT335 (Wolff et al., 1992; Bobinnec et al., 1998)] (Fig. $7 \mathrm{Ba}, \mathrm{b})$, and centrosomes with antibodies against $\gamma$-tubulin (Fig. 7Bc). At the level of resolution provided by immunofluorescence microscopy, no obvious defects in centrosome migration, spindle formation or chromosome segregation could be detected (Fig. 7b,c). However, a most remarkable change occurred in the relationship between centrosomes and $\mathrm{C}$-Nap1 patches. Whereas all interphase centrosomes were embedded in C-Nap1 patches (Fig. 7Aa, $\mathrm{Ba}$, none of the mitotic spindle poles seen in metaphase, anaphase or telophase cells ever showed any association with 
such patches (Fig. 7Ab,c, Bb,c, and data not shown). In contrast, C-Nap1 patches that were not in direct contact with centrosomes persisted in the cell body throughout mitosis (Fig. 7). Most interestingly, we also observed several examples of prophase cells in which one centrosome was still embedded within a C-Nap1 patch, whereas the other was already free and positioned at some distance (Fig. 7C). The one C-Nap1 patch that was still in contact with a centrosome often appeared disturbed and partially dissolved (Fig. 7C, see enlarged insets).

A

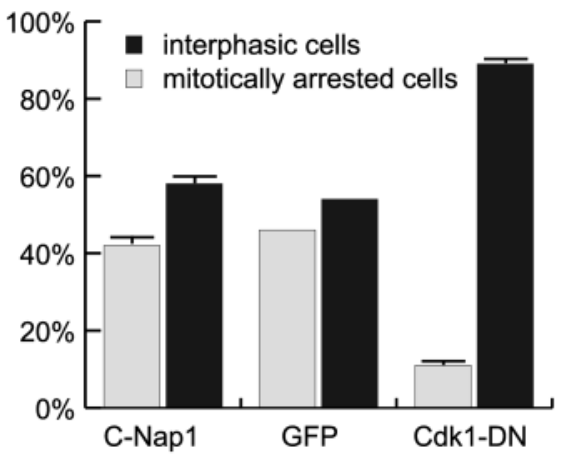

B

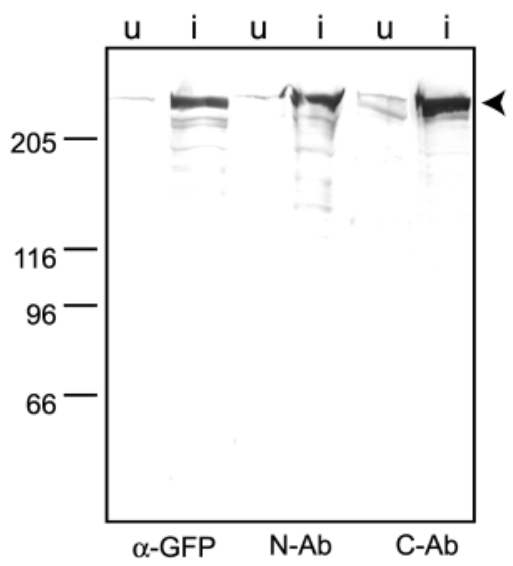

C

number

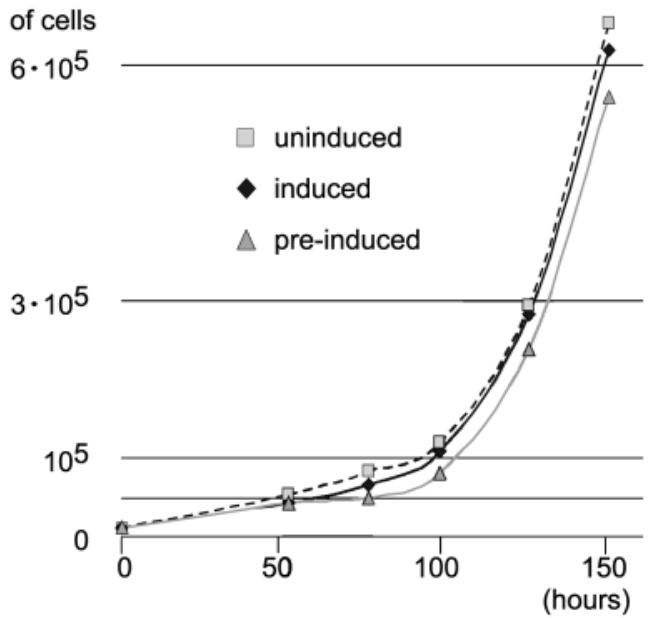
counted.
Virtually identical results were obtained when examining cells after transient overexpression of a non-tagged version of CNap1 (data not shown), indicating that the GFP marker was not responsible for the loss of C-Nap1 interaction with mitotic spindle poles. From these results we conclude that the centrosome-associated C-Nap1 patches are subject to disassembly at the very onset of mitosis, when chromosome condensation begins and centrosomes separate. Furthermore, our observations strongly argue against a generalized mechanism for the disassembly of C-Nap1-containing structures. Instead, they point to a mechanism that is activated specifically at the centrosome. As a consequence, centrosomeassociated C-Nap1 structures are locally dismantled, allowing centrosome separation and spindle formation to occur, although C-Nap1 patches elsewhere in the cell persist throughout mitosis.

\section{Active Nek2 interferes with C-Nap1 patch formation}

In previous studies, we have shown that the centrosomeassociated protein kinase Nek2 can bind and phosphorylate CNap1, both in vitro and in vivo (Fry et al., 1998b). Here, we have shown that C-Nap1 is almost certainly phosphorylated during $\mathrm{M}$ phase, and that overexpressed C-Nap1 is specifically removed from centrosomes at the onset of mitosis. Therefore, it was of obvious interest to ask whether co-expression of Nek2 would influence the formation or stability of C-Nap1 patches. To this end, C-Nap1 was co-expressed with either wild-type Nek2 or a catalytically inactive Nek2 mutant. Then, cells were scored for the frequency and appearance of C-Nap1 patches (Fig. 8A). To facilitate data analysis, transfected cells were grouped into three classes, depending on the observed C-Nap1 distribution (Fig. 8B). The first class comprised cells showing little, if any, unusual C-Nap1 structures (Fig. 8Ba). In the second class, we counted cells with one or few $(<10)$ large patches (Fig. 8Bb), and in the third class cells harboring many, often small C-Nap1-positive patches (>10) or speckles (Fig. $8 \mathrm{Bc})$. Using this classification, we observed a very striking effect of active, but not inactive Nek2, on the formation of CNap1-positive structures. Cells expressing the catalytically inactive Nek2 mutant in fact contained predominantly class II cells with few but large C-Nap1 patches (Fig. 8A), as observed for cells transfected with C-Nap1 only (see above). In contrast,

Fig. 6. C-Nap1 overexpression does not prevent cell cycle progression. (A) U2OS cells were co-transfected with two plasmids, one encoding a nondestructible cyclin B2 mutant and the second C-Nap1, GFP or Cdk1-DN, as indicated. At 36 hours post-transfection, cells were fixed and the percentages of cells in interphase (black bars) and mitosis (light gray bars) determined. Transfected cells were detected using the 9E10 mAb for C-Nap1 and anti-HA $\mathrm{mAb}$ for Cdk1-DN. Enhanced GFP was detected using the FITC filter. In two independent experiments, more than 200 cells were counted. (B) Integration and expression of GFP-C-Nap1 in TREx modified U2OS cells. Expression of the fusion protein was induced for 24 hours with $1 \mu \mathrm{g} / \mathrm{ml}$ tetracycline. Induced (i) or uninduced $(\mathrm{u})$ cells were lysed directly in sample buffer, and extracts were analyzed by immunoblotting using the indicated antibodies. The positions of molecular weight markers are indicated (in $\mathrm{kDa}$ ) and the arrowhead marks CNap1. (C) Growth rates were determined for stable cell lines in which the expression of GFP-C-Nap1 had not been induced (uninduced), or induced (1 $\mu \mathrm{g} / \mathrm{ml}$ tetracycline) at either time 0 (induced) or 24 hours before the initiation of the experiment (pre-induced). Cells were collected at the times indicated and 
the presence of active Nek2 strikingly diminished the frequency of cells with large C-Nap1 structures, and instead, most cells now fell into class III, showing a multitude of small C-Nap1-positive speckles (Fig. 8A). We interpret this observation to indicate that $\mathrm{Nek} 2$ kinase activity interfered with the formation of large C-Nap1 aggregates, thereby favoring the formation of a multitude of small C-Nap1 structures. Although obtained in an artificial experimental setting, these results support the view that C-Nap1 is an in vivo substrate of Nek2, and that Nek2 activity may thus be responsible for the dissociation of C-Nap1 from mitotic spindle poles.

A
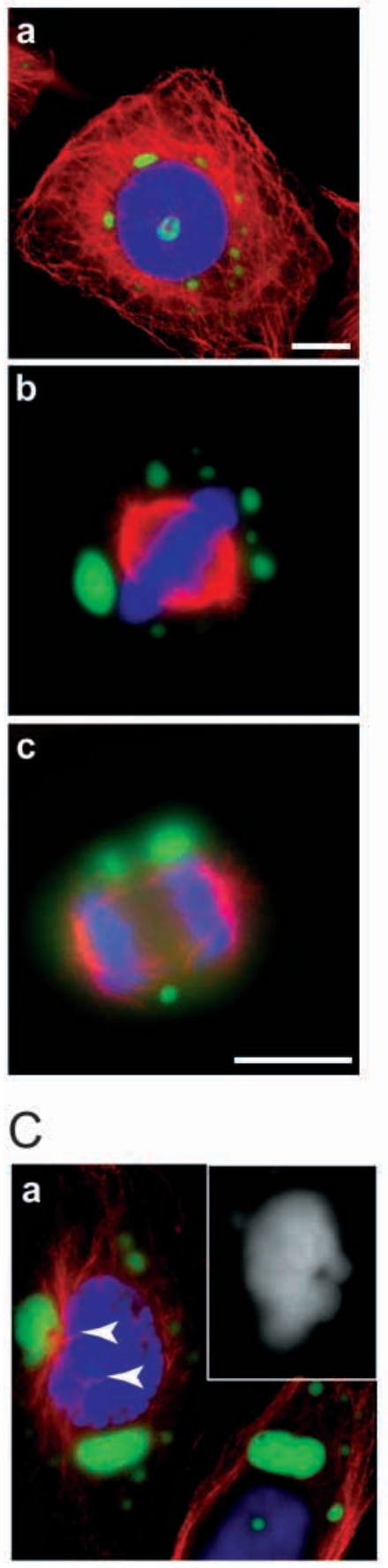
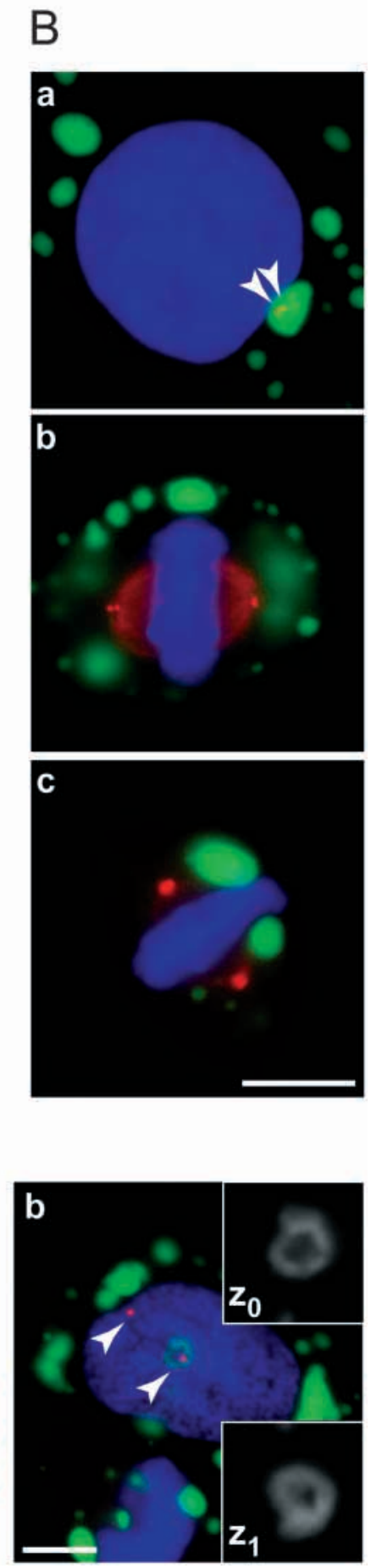

\section{Discussion}

C-Nap1 is phosphorylated during mitosis, not degraded

In the first part of the present study, we have examined the fate of C-Nap1 protein upon entry of cells into mitosis. In particular, we have sought evidence to suggest an involvement of either proteolysis or phosphorylation in the dissociation of C-Nap1 from mitotic centrosomes. From western blotting data, it appeared that total C-Nap1 protein levels were diminished by as much as $30-40 \%$ in mitotic extracts, when compared with levels in interphasic extracts. However, inhibition of the proteasome did not prevent this apparent reduction in C-Nap1 levels, arguing against an involvement of ubiquitin-dependent proteolysis. Furthermore, C-Nap1 was stable in Xenopus Mphase extracts, and no specific cleavage products could be observed. Thus, at the present time there is no evidence to support a role for proteolysis in the regulation of C-Nap1 levels. Instead, our data suggest that C-Nap1 dissociates from centrosomes as a result of phosphorylation. First, when CNap1 was immunoprecipitated from M-phase extracts and then treated with alkaline phosphatase prior to western blotting, the signal intensity was restored to the levels seen for interphasic C-Nap1. It seems most likely that the observed reduction in signal intensity was due to a reduced efficiency in the transfer of phosphorylated C-Nap1 to nitrocellulose membranes, as similar reductions in signal intensity were also seen upon phosphorylation of recombinant C-Nap1 by the Nek2 protein kinase (T.M. and E.A.N., unpublished). Second, direct in vivo labeling studies with $\left[{ }^{32} \mathrm{P}\right]$ indicate that endogenous C-Nap1 is phosphorylated in a cell-cycle-regulated manner, with maximal phosphorylation occurring during $M$ phase. Therefore, we conclude that the dissociation of C-Nap1 from mitotic centrosomes is most likely triggered by $\mathrm{C}-\mathrm{Nap} 1$ phosphorylation rather than proteolysis.

\section{C-Nap1 can self-assemble into large structures}

In a second, complementary approach, we have examined the consequences of overexpressing C-Nap1 in human cells. The main purpose of these experiments was to ask whether deregulated expression of C-Nap1 would interfere with centrosome separation at the onset of mitosis, and thus possibly block mitotic progression. We found that exogenous C-Nap1 formed large structures (termed patches) within the cell cytoplasm. The first of these patches invariably assembled around the centrosome, but with increasing C-Nap1 levels,

Fig. 7. Cells overexpressing GFP-C-Nap1 establish functional mitotic spindles. (A) GFP-C-Nap1 expression was induced for 24 hours and cells were stained for MTs in red (anti- $\alpha$-tubulin mAb), DNA in blue (Hoechst dye) and C-Nap1 in green (GFP fluorescence). Representative cells are shown in interphase (a), metaphase (b) and anaphase (c). Bars, $10 \mu \mathrm{m}$. (B) Further immunofluorescent analysis of GFP-C-Nap1-expressing cells. Staining in red shows poly-glutamylated tubulin that predominantly localizes to centrioles (GT335; a,b) and spindle poles (anti- $\gamma$-tubulin; c); staining in blue shows DNA and in green shows C-Nap1. Shown are representative cells in interphase (a) and mitosis (b,c). Bar, 10 $\mu \mathrm{m}$. (C) Prophase cells were stained in red for $\alpha$-tubulin (a) and $\gamma$ tubulin (b), in blue for DNA and in green for C-Nap1. Enlarged $(2 \times)$ insets of the C-Nap1 patches surrounding the centrosome are shown in black and white (b); $\mathrm{z}_{0}$ and $\mathrm{z}_{1}$ designate two different focal planes. Bar, $10 \mu \mathrm{m}$. 
A

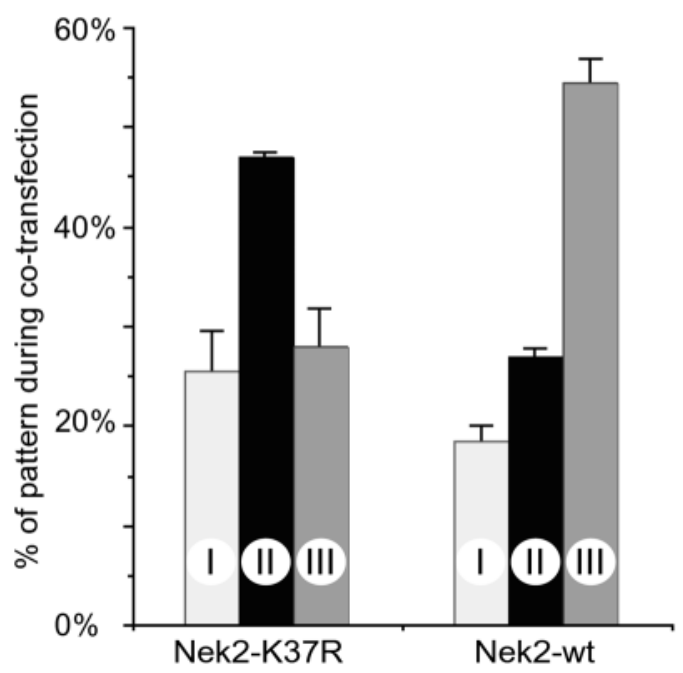

B

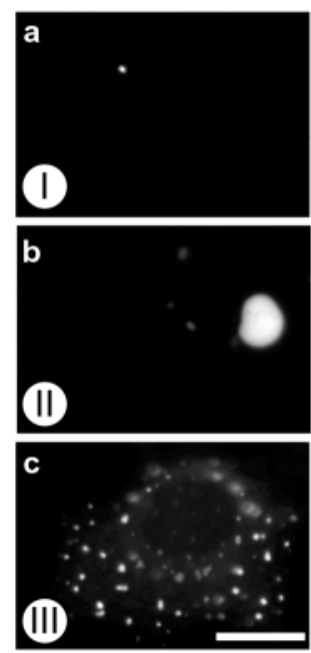

Fig. 8. Active Nek2 modulates C-Nap1 patch formation. (A) Histograms illustrate the percentage of transfected cells falling into three defined categories (as defined in B), after co-transfection of mycC-Nap1 with either active Nek2 (Nek2-wt) or a catalytically inactive Nek2 mutant (Nek2-K37R). Results are shown as averages of two independent experiments. (A total of 588 cells were counted for co-transfections with Nek2-K37R and 600 cells for co-transfections with Nek2-wt.) (B) Representative examples of three types of C-Nap1 structures, used for classification of transfected cells. Class I, CNap1 localized to centrosomes without inducing the formation of grossly abnormal structures (a). Class II, C-Nap1 formed one predominant patch closely associated with the centrosome, with or without accompanying smaller patches $(<10$ patches in total) (b). Class III, C-Nap1 formed many ( $>10)$, often small, patches (speckles) (c). Bar, $10 \mu \mathrm{m}$ (Ac). additional patches formed throughout the cytoplasm. Of several centrosome-associated proteins examined ( $\mathrm{Nek} 2, \gamma-$ tubulin, centrin-2, Plk1, p150 Glued), only Nek2 was recruited to a significant extent to the C-Nap1 patches. This underscores the affinity of Nek2 for C-Nap1, but also suggests that ectopically expressed C-Nap1 interacts largely with itself, recruiting at most a limited subset of centrosomal proteins into the patches. As shown by a MT re-growth assay, C-Nap1 patches interfered with MT-nucleation activity. We believe that this reflects steric hindrance rather than a direct role of C-Nap1 in MT-nucleation. Conversely, no increased MT-nucleation activity could be observed, in line with the view that C-Nap1 plays a structural role at the centrosome.

Although the C-Nap1 patches described here clearly represent non-physiological structures, they are reminiscent of aberrant centrosome-related structures that occur in human tumors. Centrosomal aberrations have long been described in human cancers (Boveri, 1914), and more recently were shown to include supernumerary centrioles, excess pericentriolar material and fragmented centrosomal structures (for reviews, see Lingle and Salisbury, 2000; Brinkley, 2001; Duensing and Munger, 2001; Doxsey, 2001). If C-Nap1 overexpression were to occur under pathological conditions, our present results predict that this would probably not affect cell division. However, considering that centrosomes play an important role in organizing the microtubule network also during interphase of the cell cycle, it would be premature to exclude that aberrant centrosomal structures formed by C-Nap1 and other structural proteins could interfere with MT-dependent processes such as cell shape, polarity or motility.

\section{C-Nap1 patches are dismantled around centrosomes during prophase}

Considering the extensive embedding of centrosomes into CNap1 patches, and the concomitant suppression of MT nucleation, we were surprised to find that C-Nap1 patches did not detectably interfere with cell proliferation. However, detailed examination of the fate of $\mathrm{C}$-Nap1 patches during the cell cycle offered an explanation for this apparent paradox: staining of centrosomes with appropriate antibodies in fact revealed that centrosomes are extracted from the embrace of C-Nap1 patches during early prophase. As a result, bipolar spindles readily formed, and spindle poles were never embedded within C-Nap1 patches. In striking contrast, C-Nap1 patches that were not in direct contact with centrosomes persisted throughout cell division. These results strongly suggest that centrosomes harbor an activity that, at the onset of mitosis, can trigger the local disassembly of C-Nap1. As suggested previously (Fry et al., 1998a), the protein kinase Nek2 is a prime candidate for providing such an activity. This notion was further corroborated here by our demonstration that co-expression of Nek2 with C-Nap1 profoundly affected the formation of C-Nap1 patches, strongly indicating that Nek2 is able to phosphorylate C-Nap1.

Although attractive, the idea that Nek2 triggers C-Nap1 dissociation from centrosomes is confounded by two problems: first, as we have shown here, Nek2 is recruited to all C-Nap1 patches, and yet only the centrosome-associated patch disassembles at the onset of mitosis. Hence, another centrosome-associated activity may be limiting for C-Nap1 disassembly. Alternatively, our observations could be explained if the Nek2 antagonist PP1 was also recruited to CNap1 patches, but only inactivated by $\mathrm{Cdk} 1 /$ cyclin $\mathrm{B}$ at the centrosome. Although we presently have no appropriate antibodies to test whether PP1 is recruited to C-Nap1 patches, the observed centrosome-association of Cdk1/cyclin B (Bailly et al., 1992) would be consistent with such a scenario. A second problem stems from the previous finding that Nek2 protein and activity levels are strongly reduced in nocodazole arrested cells (Fry et al., 1995). At first glance, this would seem to argue against Nek2 being the kinase responsible for phosphorylating C-Nap1 in the experiments described here. However, we emphasize that recent studies have revealed the existence of two alternatively spliced forms of Nek2, Nek2A and Nek2B, not only in Xenopus (Fry et al., 2000; Uto and Sagata, 2000) but also in human cells (Hames et al., 2001). Although Nek2A is degraded in nocodazole-arrested cells, due 
to ubiquitin-dependent proteolysis, Nek2B is stable (Hames et al., 2001). Thus, this newly discovered, shorter form of the Nek2 kinase could readily continue to phosphorylate C-Nap1 even after the destruction of Nek2A. To definitively prove that Nek2A and/or B is the kinase responsible for C-Nap1 disassembly, it will be necessary to identify the in vivo phosphorylation sites within C-Nap1 and compare them with the sites phosphorylated by Nek2 in vitro. So far, all our attempts at identifying in vivo phosphorylation sites within CNap1 have been unsuccessful, at least in part due to low protein abundance. However, with the increasing sensitivity of mass spectrometry, it seems legitimate to hope that this goal may come within reach in the not too distant future.

We thank Olaf Kelm for providing Xenopus extracts and all colleagues in our laboratory for discussions. In its initial stage, this work was supported by the Swiss National Science Foundation (3150576.97) and the Canton of Geneva.

\section{References}

Bailly, E., Pines, J., Hunter, T. and Bornens, M. (1992). Cytoplasmic accumulation of cyclin B1 in human cells: association with a detergentresistant compartment and with the centrosome. J. Cell Sci. 101, 529-545.

Bobinnec, Y., Moudjou, M., Fouquet, J. P., Desbruyeres, E., Edde, B. and Bornens, M. (1998). Glutamylation of centriole and cytoplasmic tubulin in proliferating non-neuronal cells. Cell Motil. Cytoskeleton 39, 223-232.

Boveri. T. (1914). Zur Frage der Entstehung maligner Tumoren. Jena: Fischer Verlag.

Brinkley, B. R. (2001). Managing the centrosome numbers game: from chaos to stability in cancer cell division. Trends. Cell Biol. 11, 18-21.

Doxsey, S. (2001). Re-evaluating centrosome function. Nat. Rev. Mol. Cell Biol. 2, 688-698.

Duensing, S. and Munger, K. (2001). Centrosome abnormalities, genomic instability and carcinogenic progression. Biochim. Biophys. Acta. 1471, M81-M88.

Fabunmi, R. P., Wigley, W. C., Thomas, P. J. and DeMartino, G. N. (2000). Activity and regulation of the centrosome-associated proteasome. J. Biol. Chem. 275, 409-413.

Freed, E., Lacey, K. R., Huie, P., Lyapina, S. A., Deshaies, R. J., Stearns, T. and Jackson, P. K. (1999). Components of an SCF ubiquitin ligase localize to the centrosome and regulate the centrosome duplication cycle. Genes Dev. 13, 2242-2257.

Fry, A. M., Schultz, S. J., Bartek, J. and Nigg, E. A. (1995). Substrate specificity and cell cycle regulation of the Nek2 protein kinase, a potential human homolog of the mitotic regulator NIMA of Aspergillus nidulans. $J$. Biol. Chem. 270, 12899-12905.

Fry, A. M., Mayor, T., Meraldi, P., Stierhof, Y. D., Tanaka, K. and Nigg, E. A. (1998a). C-Nap1, a novel centrosomal coiled-coil protein and candidate substrate of the cell cycle-regulated protein kinase Nek2. J. Cell Biol. 141, 1563-1574.

Fry, A. M., Meraldi, P. and Nigg, E. A. (1998b). A centrosomal function for the human Nek2 protein kinase, a member of the NIMA family of cell cycle regulators. EMBO J. 17, 470-481.

Fry, A. M., Descombes, P., Twomey, C., Bacchieri, R. and Nigg, E. A. (2000). The NIMA-related kinase $\mathrm{X}-\mathrm{Nek} 2 \mathrm{~B}$ is required for efficient assembly of the zygotic centrosome in Xenopus laevis. J. Cell Sci. 113, 1973-1984.

Gallant, P. and Nigg, E. A. (1992). Cyclin B2 undergoes cell cycle-dependent nuclear translocation and, when expressed as a non-destructible mutant, causes mitotic arrest in HeLa cells. J. Cell Biol. 117, 213-224.

Gstaiger, M., Marti, A. and Krek, W. (1999). Association of human SCF(SKP2) subunit p19(SKP1) with interphase centrosomes and mitotic spindle poles. Exp. Cell Res. 247, 554-562.

Hames, R. S., Wattam, S. L., Yamano, H., Bacchieri, R. and Fry, A. M. (2001). APC/C-mediated destruction of the centrosomal kinase Nek2A occurs in early mitosis and depends upon a cyclin A-type D-box. EMBO J. 20, 7117-7127.

Helps, N. R., Luo, X., Barker, H. M. and Cohen, P. T. (2000). NIMA-related kinase 2 (Nek2), a cell-cycle-regulated protein kinase localized to centrosomes, is complexed to protein phosphatase 1. Biochem. J. 349, 509518.

Kapoor, T. M., Mayer, T. U., Coughlin, M. L. and Mitchison, T. J. (2000). Probing spindle assembly mechanisms with monastrol, a small molecule inhibitor of the mitotic kinesin, Eg5. J. Cell Biol. 150, 975-988.

Lingle, W. L. and Salisbury, J. L. (2000). The role of the centrosome in the development of malignant tumors. Curr. Top. Dev. Biol. 49, 313-329.

Mack, G. J., Rees, J., Sandblom, O., Balczon, R., Fritzler, M. J. and Rattner, J. B. (1998). Autoantibodies to a group of centrosomal proteins in human autoimmune sera reactive with the centrosome. Arthritis Rheum. 41, 551-558.

Mayer, T. U., Kapoor, T. M., Haggarty, S. J., King, R. W., Schreiber, S. L. and Mitchison, T. J. (1999). Small molecule inhibitor of mitotic spindle bipolarity identified in a phenotype-based screen. Science 286, 971-974.

Mayor, T., Meraldi, P., Stierhof, Y. D., Nigg, E. A. and Fry, A. M. (1999). Protein kinases in control of the centrosome cycle. FEBS Lett. 452, 92-95.

Mayor, T., Stierhof, Y. D., Tanaka, K., Fry, A. M. and Nigg, E. A. (2000). The centrosomal protein C-Nap1 is required for cell cycle-regulated centrosome cohesion. J. Cell Biol. 151, 837-846.

Meraldi, P. and Nigg, E. A. (2001). Centrosome cohesion is regulated by a balance of kinase and phosphatase activities. J. Cell Sci. 114, 37493757.

Paintrand, M., Moudjou, M., Delacroix, H. and Bornens, M. (1992). Centrosome organization and centriole architecture: their sensitivity to divalent cations. J. Struct. Biol. 108, 107-128.

Puntoni, F. and Villa-Moruzzi, E. (1997). Protein phosphatase-1 alpha, gamma 1, and delta: changes in phosphorylation and activity in mitotic HeLa cells and in cells released from the mitotic block. Arch. Biochem. Biophys. 340, 177-184.

Schultz, S. J., Fry, A. M., Sutterlin, C., Ried, T. and Nigg, E. A. (1994). Cell cycle-dependent expression of Nek2, a novel human protein kinase related to the NIMA mitotic regulator of Aspergillus nidulans. Cell Growth Differ. 5, 625-635.

Seelos, C. (1997). A critical parameter determining the aging of DNAcalcium-phosphate precipitates. Anal. Biochem. 245, 109-111.

Uto, K. and Sagata, N. (2000). Nek2B, a novel maternal form of Nek2 kinase, is essential for the assembly or maintenance of centrosomes in early Xenopus embryos. EMBO J. 19, 1816-1826.

van den Heuvel, S. and Harlow, E. (1993). Distinct roles for cyclindependent kinases in cell cycle control. Science 262, 2050-2054.

Vorlaufer, E. and Peters, J. M. (1998). Regulation of the cyclin B degradation system by an inhibitor of mitotic proteolysis. Mol. Biol. Cell 9, 1817-1831.

Wolff, A., de Nechaud, B., Chillet, D., Mazarguil, H., Desbruyeres, E., Audebert, S., Edde, B., Gros, F. and Denoulet, P. (1992). Distribution of glutamylated alpha and beta-tubulin in mouse tissues using a specific monoclonal antibody, GT335. Eur. J. Cell Biol. 59, 425-432. 